\title{
The challenge of evolving stable polyploidy: could an increase in "crossover interference distance" play a central role?
}

\author{
Kirsten Bomblies $^{1} \cdot$ Gareth Jones $^{2}$ • Chris Franklin ${ }^{3}$ - Denise Zickler ${ }^{4} \cdot$ Nancy Kleckner ${ }^{5}$
}

Received: 10 June 2015 /Revised: 20 December 2015 / Accepted: 28 December 2015 /Published online: 12 January 2016

(C) The Author(s) 2016. This article is published with open access at Springerlink.com

\begin{abstract}
Whole genome duplication is a prominent feature of many highly evolved organisms, especially plants. When duplications occur within species, they yield genomes comprising multiple identical or very similar copies of each chromosome ("autopolyploids"). Such genomes face special challenges during meiosis, the specialized cellular program that underlies gamete formation for sexual reproduction. Comparisons between newly formed (neo)-autotetraploids
\end{abstract}

This article is part of a Special Issue on "Recent advances in meiotic chromosome structure, recombination and segregation" edited by Marco Barchi, Paula Cohen and Scott Keeney.

Electronic supplementary material The online version of this article (doi:10.1007/s00412-015-0571-4) contains supplementary material, which is available to authorized users.

Nancy Kleckner

kleckner@fas.harvard.edu

Kirsten Bomblies

Kirsten.Bomblies@jic.ac.uk

Chris Franklin

Franklin@bham.ac.uk

Denise Zickler

denise.zickler@i2bc.paris-saclay.fr

1 Department of Cell and Developmental Biology, John Innes Centre, Norwich Research Park, Colney, Norwich NR4 7UH, UK

2 The Red House, St. David's Street, Presteigne, Powys (Wales) LD8 2BP, UK

3 School of Biosciences, University of Birmingham, Edgbaston, Birmingham B15 2TT, UK

4 Institut de Génétique et Microbiologie, I2BC, Université Paris-Sud, Orsay, France

5 Department of Molecular and Cellular Biology, Harvard University, Cambridge, MA, USA and fully evolved autotetraploids suggest that these challenges are solved by specific restrictions on the positions of crossover recombination events and, thus, the positions of chiasmata, which govern the segregation of homologs at the first meiotic division. We propose that a critical feature in the evolution of these more effective chiasma patterns is an increase in the effective distance of meiotic crossover interference, which plays a central role in crossover positioning. We discuss the findings in several organisms, including the recent identification of relevant genes in Arabidopsis arenosa, that support this hypothesis.

Keywords Polyploidy $\cdot$ Meiosis $\cdot$ Crossover interference $\cdot$ Homologous chromosomes $\cdot$ Recombination $\cdot$ Chiasmata

\section{Introduction}

Most eukaryotic organisms have diploid genomes. However, in some cases, a genome contains more than two homologous copies of each chromosome. This condition, known as "polyploidy," occurs in many species, notably plants but also many animals (Astaurov 1969; Ramsey and Schemske 1998; Otto and Whitton 2000; Ramsey and Schemske 2002; Comai 2005; Soltis et al. 2007; Doyle et al. 2008; Grandont et al. 2013; Stenberg and Saura 2013; Ianzini et al. 2009). Polyploidy is a potent evolutionary force that is implicated in increases of genome complexity, adaptation, and speciation (Soltis et al. 2003; Rieseberg and Willis 2007; Fawcett and Van de Peer 2010; Arrigo and Barker 2012). However, when the polyploid condition first arises, it causes substantial problems for basic cellular processes, most notably for regular chromosome segregation during meiosis, the specialized cellular program that underlies gamete formation for sexual reproduction (Ramsey and Schemske 2002; Comai 2005; 
Stenberg and Saura 2013; below). Nonetheless, this problem can ultimately be resolved: stable, sexually reproducing polyploid lineages are found in nature and sometimes can be generated in experimental settings upon selection for fertility. How these states evolve remains largely mysterious, and investigation of this question promises intriguing insights into how fundamental processes can be evolutionarily modified without perturbing their essential functions.

Polyploids generally fall into two broad categories, albeit with a range of intermediate states (Ramsey and Schemske 1998; Otto and Whitton 2000; Bomblies and Madlung 2014). "Autopolyploids" arise by whole genome duplication within an individual or within a species, yielding genome complements in which all copies of a particular chromosome are closely homologous (although still carrying sequence differences present between homologs in the originating diploid). "Allopolyploids," in contrast, arise by formation of hybrids with related yet distinct genomes, accompanied by whole genome duplication. The component genomes of allopolyploids thus comprise two or more sets of diploid genomes, with a closer homology between the two chromosomes of each set and a significantly less homology between chromosomes of different sets.

In diploid meiosis, homologs regularly segregate away from one another, to opposite poles, at the first division of meiosis (MI); sister chromatids then segregate at the second division of meiosis (MII). For full fertility in polyploid meiosis, the MI must still somehow result in a regular segregation of equal numbers of parental chromosomes to each pole, despite the fact that there are now multiple copies of each chromosome present. This challenge is particularly stark for autopolyploids, because the different versions of each chromosome are very similar or indistinguishable in DNA sequence and thus provide no special intrinsic cues to guide equipartitioning during the segregation process. For allopolyploids, in contrast, the presence of homology can be (and is) used to solve this problem, as shown by the fact that more homologous chromosomes preferentially segregate from one another (e.g., Holm 1986; discussion in Zickler and Kleckner 1999; Bhullar et al. 2014; Martín et al. 2014). We focus here on the more dramatic case of autopolyploidy, which has been investigated extensively from various perspectives.

\section{Newly arising autotetraploids evolve to a stable fertile state via restrictions on the number and types of chiasma configurations}

Newly emerged autopolyploid lines generated in the laboratory or arising in nature generally exhibit meiotic chromosome mis-segregation in meiosis I, resulting in the formation of aneuploid gametes and compromised fertility (Ramsey and Schemske 1998, 2002; Hilpert 1957; Santos et al. 2003;
Comai 2005; Grandont et al. 2013; Sybenga 1975). However, naturally occurring polyploids can evolve solutions to overcome these problems.

\section{Diploid meiosis}

To contextualize the challenges faced by polyploids in meiosis, we first discuss the normal process that occurs in diploids.

In a diploid cell at metaphase I, the centromeres of homologous chromosomes (homologs) are oriented towards opposite poles, in preparation for segregation during the ensuing anaphase (Fig. 1(a, b)). At this stage, homologs are connected to one another at specific positions, seen cytologically as one or more "chiasmata," each of which results from the combined effects of a DNA crossover (CO) between homolog non-sister chromatids plus links between sister chromatid arms along their lengths (Fig. 1(a, b)).

These connections are essential for the regular segregation of homologs to opposite poles, analogous to the way in which a physical connection of sister chromatids ensures bipolar orientation and segregation during mitosis and metaphase/ anaphase of the second meiotic division ( $\mathrm{Li}$ and Nicklas 1997; Nicklas 1997). In all cases, connectedness is important because it allows centromere/kinetochore complexes to be placed under mechanical tension as the spindle is forming. This tension, in turn, has two effects which, together, ensure bipolar orientation of the segregating units ( $\mathrm{Li}$ and Nicklas 1997; Nicklas 1997; Lampson and Cheeseman 2011). First, tension stabilizes microtubule/kinetochore attachments, thus locking each bivalent into the correct configuration. Second, tension signals to the spindle regulatory surveillance system (the "spindle assembly checkpoint") that a bivalent is properly aligned and thus ready to be correctly pulled towards opposite poles during anaphase I. When all segregating pairs (e.g., meiotic homolog "bivalents") are correctly oriented to opposite poles, cellular regulatory mechanisms trigger anaphase onset. In the absence of correct orientation, anaphase eventually proceeds but with a significant delay.

In accordance with these considerations, a first requirement for regular MI segregation is that each pair of homologs must (and does) acquire at least one CO (and thus chiasma). This feature is often called the "obligatory CO." Many diploid species exhibit only one or two COs/chiasmata per bivalent (e.g., Arabidopsis thaliana and Arabidopsis arenosa; Fig. 1(a, b)); others exhibit larger numbers.

A second requirement for regular MI segregation is that each bivalent must be a separate physical unit that is free of entanglements with other bivalents. In fact, interlockings among unrelated chromosomes often arise during prophase as a consequence of the way in which homologs undergo recombination while becoming coaligned/paired and synapsed. However, interlocks are also concomitantly actively 


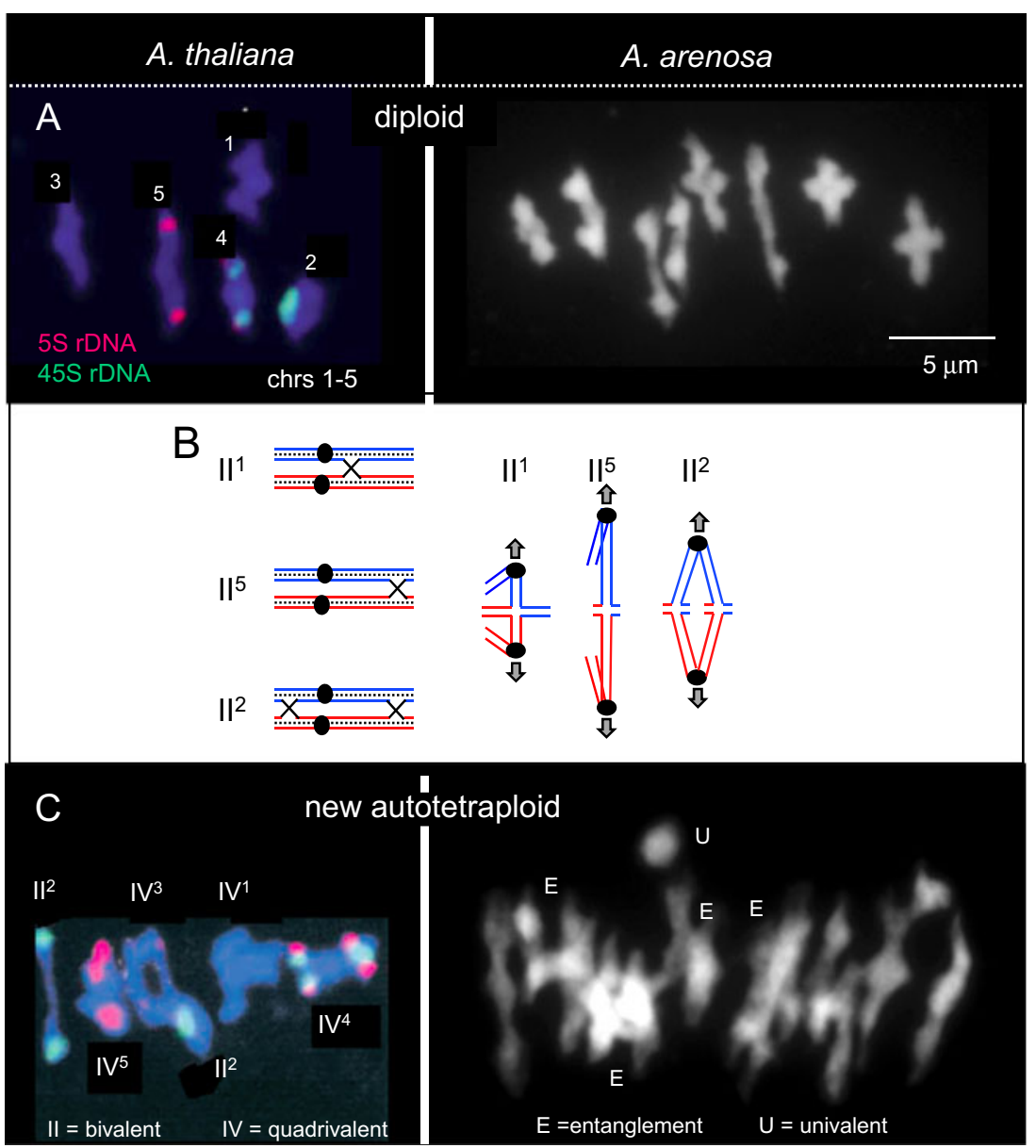

Fig. 1 Metaphase I (MI) configurations in diploids and newly arisen autotetraploids of Arabidopsis. Chromosomes are being pulled towards opposite spindle poles (above and below, respectively) via attachments of microtubules to the respective centromere/kinetochore regions. a (Left) Arabidopsis thaliana diploid showing five bivalents (from López et al. 2012). The chromosome number of each bivalent is indicated. $45 \mathrm{~S}$ and $5 \mathrm{~S}$ rDNA loci are indicated. a (Right) Arabidopsis arenosa diploid showing eight bivalents (C.F. and C. Morgan, unpublished). $b$ Cartoons showing chromosome associations that give rise to three of the metaphase I

eliminated (Storlazzi et al. 2010). Thus, at MI, interlocked bivalents are rare or absent.

\section{Meiosis in neo-autotetraploids}

A newly arisen autotetraploid line faces severe challenges during MI chromosome segregation. Since all four copies of each chromosome are essentially the same, COs can (and do) occur indiscriminately among these copies in all pairwise combinations. This situation compromises both of the features required for regular two-by-two segregation.

First, newly arising autotetraploids exhibit complex arrays of chiasma configurations (e.g., Fig. 1(c)). The four chromosome copies may happen to form two pairs of bivalents that will give regular two-by-two segregation. However, they can configurations seen in $a$ (left). The arrows indicate the orientation of centromeres (filled circles) towards opposite spindle poles. II denotes the bivalent; superscript denotes the chromosome number. $c$ (Left) An experimentally created autotetraploid of $A$. thaliana showing a mixture of bivalents (II) and quadrivalents (IV) (from Santos et al. 2003). c (Right) An experimentally created autotetraploid of $A$. arenosa showing some identifiable bivalents, many complex configurations in which multiple chromosomes are entangled $(E)$ and one apparent univalent $(U)$ (Chris Morgan and C. F., unpublished)

also form diverse types of multivalents (where more than two copies are connected).

Many of the configurations that arise in neo-autotetraploids are incompatible with regular two-by-two segregation. Overall, the operative rule for effective two-by-two segregation is that every one of the four homologous chromosomes must be linked to at least one, but not more than two, partner chromosome(s) (Fig. 2). Sets of linkages that satisfy this rule comprise bivalents and either chain or ring quadrivalents (Fig. 2a). In all three cases, spindle tension is achieved by orientation of two centromere/ kinetochore regions towards each pole as appropriate to twoby-two segregation. In contrast, any complement that includes a chromosome(s) unlinked to any partner will be ineffective (e.g., a univalent-plus-trivalent combination; Fig. 2b, left). Additionally, in any complement where one or more 


\section{Auto-tetraploid Chiasma Configurations}

A Effective
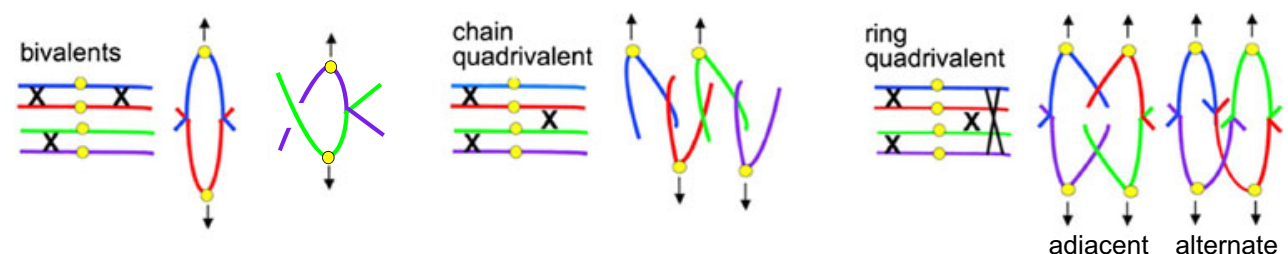

\section{B Not Effective}

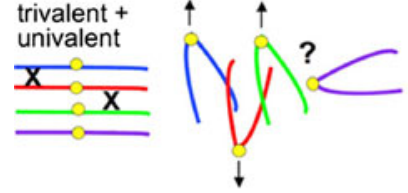

Fig. 2 Chiasma configurations for an autotetraploid that are either effective for ensuring two-by-two segregation (a) or not (b). Effective segregation requires that each chromosome be linked to either one or two other chromosomes. Only three configurations satisfy this

chromosomes are linked to each of the three other homologous chromosomes, one centromere will always be unable to come under tension and thus again will not reliably segregate to the appropriate pole (Fig. 2b, right).

Second, neo-autotetraploid chromosomes exhibit extensive numbers of chromosomal associations that appear to be comprised of both multivalents and entanglements (e.g., "E" in Fig. 1(c); see also Fig. 5 in Yant et al. 2013). These configurations are the consequence of the fact that all four homologous chromosomes often pair and synapse and form CO connections; moreover, if other homologs are trapped in those quadrivalents, they can remain entangled by their own $\mathrm{CO}$ connections up to metaphase I. In diploids, such entanglements ("interlocks") are usually resolved before diplotene/ metaphase I (von Wettstein et al. 1984). However, if they are not, the presence of entanglements is an obvious problem for MI segregation. In new autotetraploids, the presence and persistence of interlockings might primarily be responsible for the overall high mis-segregation frequencies as well for the occurrence of univalents. The resolution of interlocks requires, among other events, Mlh1-mediated release of trapped recombinational interactions (Storlazzi et al. 2010). Such a process would promote disassembly of $\mathrm{CO}$-fated recombination complexes that are stalled at sites of interlocks. Given that $\mathrm{CO}$ complexes are relatively few to begin with, the result would be a tendency for some of the entrapped chromosomes to lose all COs, thus lacking even the single "obligatory" $\mathrm{CO}$ and appearing as univalent(s) at MI.

\section{Meiosis in evolved autotetraploids}

Evolved autotetraploid species commonly show regular tetrasomic inheritance (Quiros 1982; Krebs and Hancock
1989; Wolf et al. 1989; Fjellstrom et al. 2001; Hollister et al. 2012): each locus segregates four alleles, with two of the four parental alleles randomly segregating into each gamete. Thus, evolution of an autotetraploid into a stable, sexually reproducing state has achieved regular two-by-two segregation without the emergence of specific genetically defined partner preferences. To do so, autotetraploid evolution includes changes in chiasma patterns.

First, there is a reduction in the total overall frequency of chiasmata. In general, well-evolved autotetraploids tend to have lower overall frequencies of COs than newly formed autotetraploids (e.g., Morrison and Rajhathy 1960a; Mulligan 1967; Reddi 1970; Yant et al. 2013; Wu et al. 2013). More specifically, stable autotetraploid lines that have evolved in nature have lower $\mathrm{CO}$ frequencies than the diploid lines from which they originated (Mulligan 1967; Yant et al. 2013). Furthermore, when an autotetraploid is created experimentally and then allowed to evolve under selective pressure for successful meiotic transmission, a decrease in multivalent frequency commonly occurs, associated with a reduction in the level of COs, which progressively decreases with the number of rounds of selection (e.g., Bremer and BremerReinders 1954; Povilaitis and Boyes 1956; Hilpert 1957; Morrison and Rajhathy 1960a; Lavania et al. 1991; Santos et al. 2003). In a number of species, the frequency of chiasmata is reduced to one, thus nearly the minimum possible level. For example, autotetraploids Lotus corniculatus, A. arenosa, and Physaria vitulifera all average about 1.1 crossovers per bivalent (Mulligan 1967; Davies et al. 1990; Yant et al. 2013). Lower numbers of COs/chiasmata not only match the emergence of more restricted chiasma configurations (above) but also will tend to reduce the probability of persisting MI interlockings. 
The only exception to this trend is that, in the few related grass species where autotetraploids exhibit mostly quadrivalents with terminal chiasmata (e.g., Dactylis glomerata; Table S1), the chiasma frequency is higher than that in the corresponding diploids or newly formed autotetraploids. Apparently, an evolved autotetraploid state with three or four chiasmata per quadrivalent emerged from a standard diploid condition with one or two chiasmata per bivalent.

Second, MI chiasma configuration types become more restricted, being limited specifically to those that effectively promote regular two-by-two segregation. Thus, trivalent-plusunivalent combinations are rare in naturally evolved tetraploids and, in experimental evolution studies, occur with a decreasing frequency as a stable state is progressively achieved (Povilaitis and Boyes 1956; McCollum 1958; Hazarika and Rees 1967; Jones 1967; Santos et al. 2003). Furthermore, a survey of 20 naturally evolved autopolyploid species (Table S1) reveals 12 species that form exclusively or almost exclusively bivalents at MI, with each set of four chromosomes comprising a pair of bivalents; five other species show mostly bivalents, but also some quadrivalents (e.g., A. arenosa; Fig. 3a, b), and three related species show primarily or exclusively quadrivalents. The bivalent-plus-quadrivalent solution also emerges in the laboratory when a newly formed autopolyploid evolves into a more stable line by selection for fertility over a number of generations, with improvements sometimes seen after only a few generations of selection (Gilles and Randolph 1951; Bremer and BremerReinders 1954; Hilpert 1957; Santos et al. 2003). Moreover, the quadrivalents seen in these evolved situations are either chains or rings (Fig. 3b), i.e., the two specific types that are effective for two-by-two segregation (Fig. 2a). In contrast, other possible quadrivalent configurations are rare, absent, and/or anticorrelated with regular segregation (e.g., McCollum 1958).

Ring quadrivalents can exhibit either of two MI segregation configurations according to whether "alternate" or "adjacent" centromeres are linked to the same pole (Figs. 2a and 3a). The former configuration is favored: as autotetraploidy evolves, the alternating ring configuration increases in abundance while the adjacent ring configuration decreases (McCollum 1958; Mosquin 1967). These preferences correspond to the dictate that spindle tension should be maximized: in rings of the favored alternate orientation, all four centromere/kinetochore complexes are under tension from both sides whereas, in the less-favored adjacent orientation, pairs of bi-oriented complexes are under tension from only one side.

Third, there is a tendency for modulation of chiasma position during autotetraploid evolution. There is no universal requirement for localization of chiasmata to particular positions. For example, in A. arenosa, the chiasmata in an evolved autotetraploid can occur at centromere-proximal, centromeredistal ("sub-terminal"), and interstitial positions as seen from MI configurations and confirmed by molecular analysis of

\section{A. arenosa evolved autotetraploids}
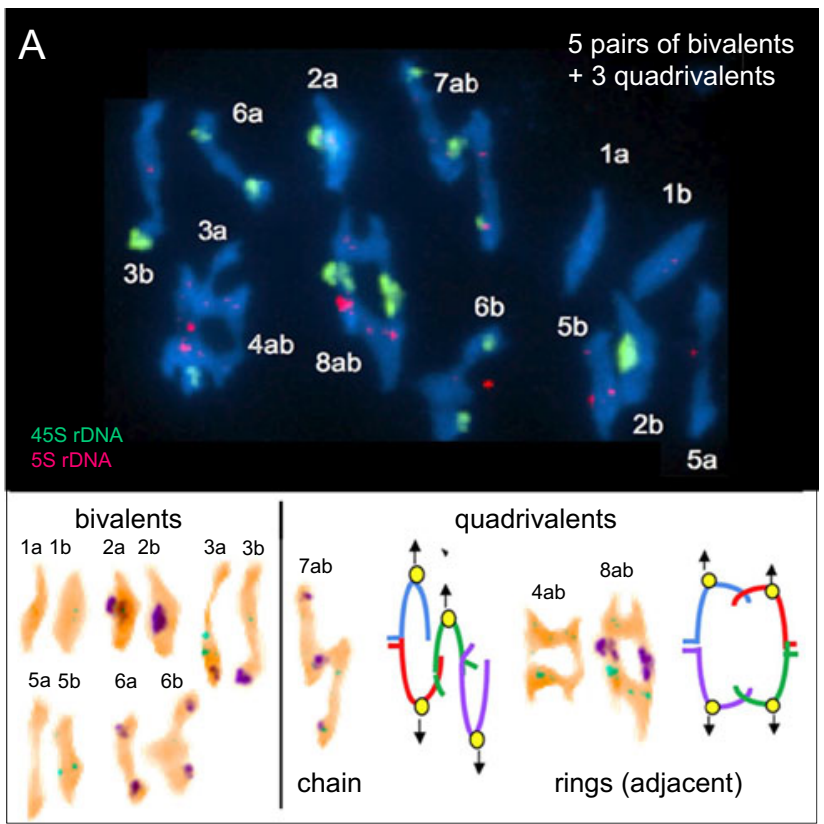

quadrivalents

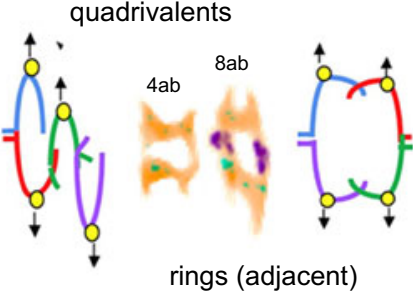

B

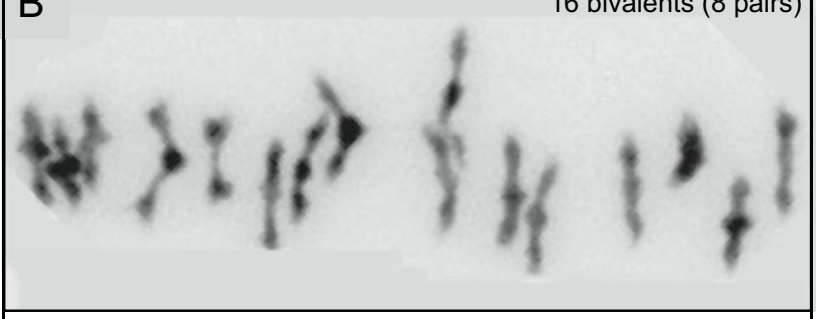

Fig. 3 Two metaphase I complements for a fully evolved autotetraploid of Arabidopsis arenosa (C. F. and C. Morgan, unpublished). a A majority of bivalents plus a minority chain and ring quadrivalents (10 bivalents corresponding to 5 pairs plus 3 quadrivalents). Accompanying colorinverted images show chromosome constitution and multivalent configurations. b Full complement of 16 bivalents corresponding to 8 pairs

CO-correlated Mlh1 foci along prophase chromosomes (Chris Morgan, C. F., and K. B., unpublished). On the other hand, the occurrence of chiasmata near chromosome ends (referred to as "terminal" localization) is positively correlated with regular quadrivalent segregation (Myers 1945; McCollum 1958; Hazarika and Rees 1967; Jones 1967), implying that such localization might be particularly helpful for creating the appropriate quadrivalent configurations. In another variation, some autotetraploid Allium species have centromere-proximal COs/chiasmata (Table S1).

Chiasmata are prominently terminal in autotetraploids of grasses and cereals (Hazarika and Rees 1967; McCollum 1958). However, the same tendency is also seen in the corresponding diploid lines. Perhaps earlier in their evolution, diploids became autotetraploids, which evolved terminal 
chiasmata, and then returned to the diploid state. Indeed, many apparently diploid genomes give evidence of prior polyploidization (e.g., Mitchell-Olds and Clauss 2002). More generally, terminal localization of chiasmata might facilitate ready interconversion between diploidy and autotetraploidy.

Fourth, evolved autotetraploids also lack the high levels of interlockings among unrelated chromosomes that characterize newly emerged lines, with resolution during pachytene as in the diploid case (compare Fig. 3a, b versus Fig. 1(c); Higgins et al. 2014a; Yant et al. 2013).

\section{Modulation of CO formation for autotetraploid evolution}

What type of mechanism(s) might explain how newly formed autotetraploids evolve the specific chiasma configurations needed to support regular two-by-two MI segregation? Since $\mathrm{CO}$ positions are determined during prophase, evolutionary forces are presumably acting on events that occur during this period, long before chiasmata are actually required to mediate chromosome alignment and segregation.

\section{CO formation in diploid meiosis}

Universally, meiosis involves the initiation of recombination via a large number of programmed double-strand breaks (DSBs) which interact primarily with homolog partners to give a large number of early recombinational interactions (Hunter 2006; Zickler and Kleckner 2015). A minority subset of these many interactions is then designated to eventually mature into COs ("CO designation") with the remainder maturing to other fates. When a bivalent exhibits more than a single $\mathrm{CO}$, those $\mathrm{COs}$ exhibit the classical feature of "crossover interference": the presence of a $\mathrm{CO}$ at one position is accompanied by a reduced probability that another $\mathrm{CO}$ will occur nearby (Sturtevant 1915). The strength of this reduction decreases with increasing interposition distance. Importantly, DSB formation and all ensuing DNA events leading to CO formation occur in recombination complexes that are in direct physical and functional association with developing or developed axes (Kleckner 2006; Kleckner et al. 2011; Zickler and Kleckner 2015; Storlazzi et al. 2010). Correspondingly, it now appears that the "metric" for the interference effect is physical distance along the chromosome, e.g., along chromosome axes, with absolute distances ranging from $300 \mathrm{~nm}$ to many microns according to the organism, rather than either genomic distance $(\mathrm{Mb})$ or genetic distance $(\mathrm{cM})$ (discussion in Zhang et al. 2014b).

We have proposed a specific model for how $\mathrm{CO}$ patterning might occur (Fig. 4a; Kleckner et al. 2004; Wang et al. 2015 and references therein). This model, which can accurately explain chiasma patterns in a variety of diploid species (Zhang et al. 2014a, b; Wang et al. 2015), has two key features. First, each CO designation sets up an "interference signal" that spreads outward in both directions from the designation site, disfavoring the occurrence of additional COs in its path. This signal is strongest at its point of origin and dissipates in strength with an increasing distance away from that starting position. Thus, a first $\mathrm{CO}$ designation will set up a surrounding zone of interference. A second $\mathrm{CO}$ designation will tend to occur outside that zone of interference. Any subsequent COs will tend to "fill in the holes" between the previously established zones, resulting in a tendency for COs to be evenly spaced, as observed (e.g., Fig. 4e).

Second, the process of $\mathrm{CO}$ designation is very efficient. As one consequence of this effect, each pair of homologs will undergo at least one $\mathrm{CO}$ designation, thus ensuring a first obligatory $\mathrm{CO}$. Thereafter, $\mathrm{CO}$ designations will continue to occur as long as there are still regions that have not been affected by interference (or, more precisely, where the effects of interference are not great enough to impede $\mathrm{CO}$ designation). Given this situation, the final array of COs will be determined by three factors: the position(s) of early recombination interactions along the chromosomes, the strength of the $\mathrm{CO}$ designation process in relation to the inhibitory effects of interference, and the distance over which $\mathrm{CO}$ interference acts. In an extreme case, where the interference distance is longer than the length of the chromosome, each homolog pair will acquire one and only one $\mathrm{CO}$, regardless of the number and positions of early recombination interactions. In diploid species, this situation occurs both genome wide (e.g., Caenorhabditis elegans; Martinez-Perez and Colaiacovo 2009; Hillers and Villeneuve 2003) and for the shorter chromosomes in the complements of several organisms, e.g., the locust Schistocerca gregaria (Fox 1973).

In most organisms, including Arabidopsis and other plants, $\mathrm{CO}$ designation and interference are thought to be implemented at a particular stage of meiosis, late leptotene, when homologs are coaligned at a distance of $\sim 400 \mathrm{~nm}$ (Fig. 4b-d, f; Zhang et al. 2015; Sanchez Moran et al. 2001; C.F., unpublished). Coalignment is affected by early recombination interactions, which, in favorable cases, can be seen as "bridges" linking the structural axes of coaligned partner chromosomes (Fig. 4b-d, f; Zickler and Kleckner 2015). Notably, however, the same conclusions will pertain as long as $\mathrm{CO}$ designation and interference operate on the array of total DSB-mediated recombination interactions, whether specifically at the "bridge stage" or not.

\section{CO formation in autotetraploids: a proposal}

The above description of meiotic prophase would suggest that in a newly formed autotetraploid, DSBs will occur on all four chromosomes and DSB-mediated bridge interactions will 
A CO-designation and Interference
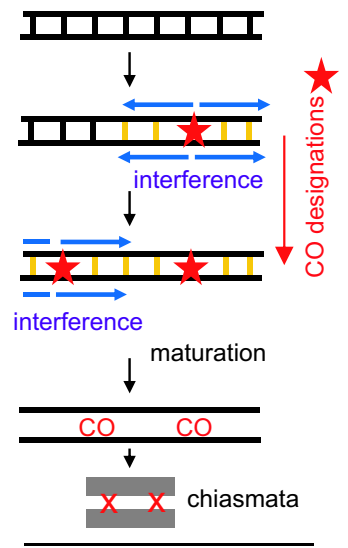

DSB-mediated inter-homolog interactions(e.g. bridges)

I before interference

after interference

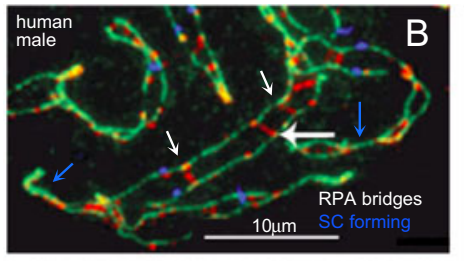

C
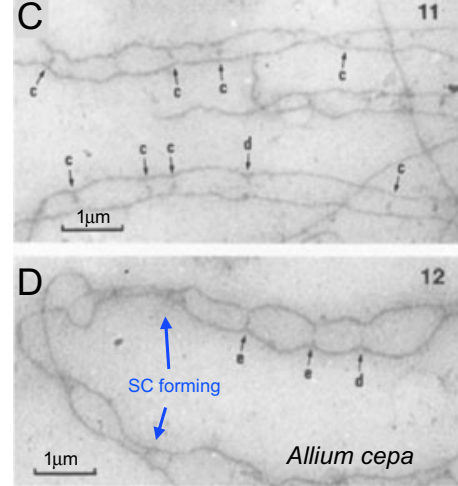

Fig. 4 Prophase chromosomal events in diploid meiosis. a "Fill-in-theholes" model for CO position selection (see also Wang et al. 2015). The array of early total DSB-mediated recombinational interactions (e.g., bridges; b-d (below)) is acted upon by a CO designation process. Each $\mathrm{CO}$ designation (red star) sets up an inhibitory zone of "CO interference" (blue arrows) via a signal that spreads outwards in both directions, dissipating with distance. This signal prevents bridge interactions in the affected region from undergoing $\mathrm{CO}$ designation (indicated by bridges changed to yellow). Subsequent $\mathrm{CO}$ designations occur in regions away from previously established interference zones, ultimately filling in the holes between previous $\mathrm{CO}$ sites. $\mathrm{CO}$ designation is very efficient, thus ensuring that all homolog pairs acquire at least one (first, obligatory) CO. b-f Homolog coalignment ("pairing") is mediated by inter-axis bridges that comprise DSB-mediated recombinational interactions and followed by SC formation ("synapsis"). b, e Human male meiotic prophase chromosomes visualized by immunofluorescence illumination. $\mathbf{c}, \mathbf{d}, \mathbf{f}$ Allium cepa axes and associated "zygotene" recombination nodules (ZNs) or bridges (corresponding approximately to many/all DSB- mediated interactions) visualized by electron microscopy of PTAstained spread preparations (from Albini and Jones 1987). b Leptotene/ zygotene nucleus illustrates bridges containing single-strand binding protein RPA (white arrows), a direct player in Rad51/Dmc1-mediated strand exchange for recombination, with accompanying onset of synapsis. Green indicates SMC3 cohesin axis, blue centromeres (blue), and red RPA protein (from Oliver-Bonet et al. 2007). c, d Bridge configurations and incipient synapsis corresponding to the stage in $\mathbf{b}$. $\mathbf{e}$ Pachytene synaptic configurations with SYCP3 axes of the SC (red), centromeres (blue), and Mlh1 foci marking sites of COs (green) (from Gruhn et al. 2013). f Two bivalents showing, respectively, extensive synapsis in progress and coalignment. In $\mathbf{c}, \mathbf{d}, \mathbf{f}$, black arrows indicate examples of "nodules" or bridges of five types: (a) associated with SCs, (b) with association sites, (c) midway between axial cores in close alignment, (d) paired structures at matching sites on axial cores, and (e) apparently bridging the space between two converging axial cores. Blue arrows and text indicate positions of forming/formed SC occur promiscuously among all possible pairs of chromosome axes. CO designation would then be imposed on this fourchromosome array. This notion is supported by tetrasomic inheritance (above) and by studies of axis relationships in autotetraploids, where coalignment of multiple axes has been observed prior to and after synaptonemal complex (SC) formation. Such complex partner interactions are common in newly formed autotetraploids (Jones and Vincent 1994; Stack and Roelofs 1996; Carvalho et al. 2010; Rasmussen 1987; Jones and Vincent 1994; Fig. 5a-e).

We suggest here that a critical event in the evolution of a fertile autotetraploid species is an increase in the "interference distance," i.e., the effective distance over which the inhibitory interference signal spreads to the point where it is comparable to, or greater than, the total lengths of the chromosomes (Fig. 6a). This required effect could be achieved by directly altering the spreading interference process per se, so that inhibition extends over a greater distance from the nucleating
$\mathrm{CO}$ site. Alternatively, given that the metric of interference is physical chromosome length (above), the equivalent effect could be achieved by shortening the physical lengths of the chromosomes: if chromosome length is decreased, a given interference distance will comprise a larger fraction of chromosome length not only in physical distance but also in genomic distance $(\mathrm{Mb})$ or genetic distance $(\mathrm{cM})$.

An increase to an appropriate (effective) interference distance will reduce the total number of COs/chiasmata. It will also specifically generate exactly the chiasma configurations observed in evolved autotetraploids. The effectiveness of this change is a direct consequence of the basic logic of the CO patterning process (above). The efficiency of $\mathrm{CO}$ designation will ensure that every chromosome copy acquires at least one $\mathrm{CO} /$ chiasma, thus eliminating "zero-CO" univalents in general and trivalent-plus-univalent configurations in particular. Concomitantly, operation of interference over a 

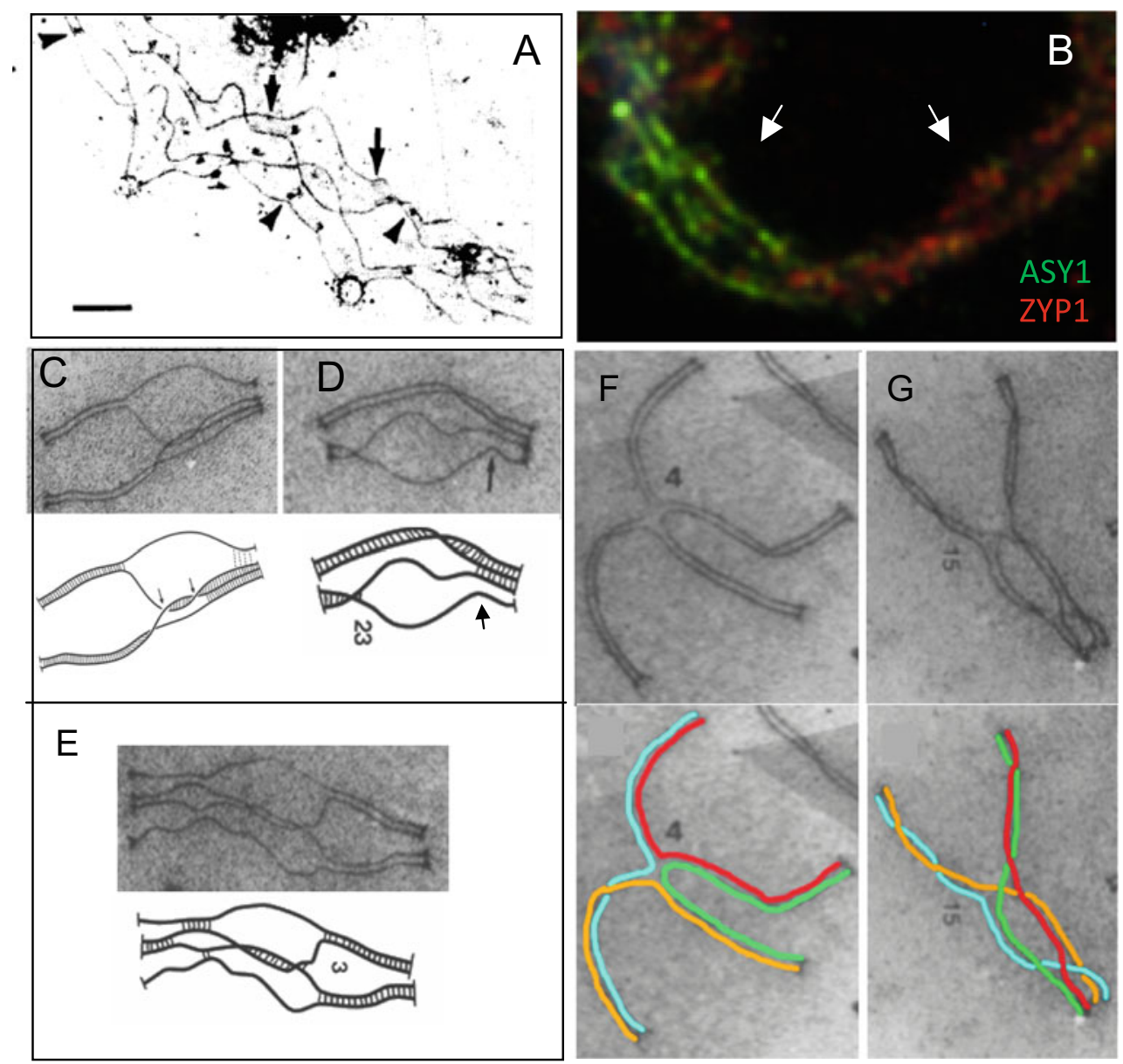

Fig. 5 Prophase relationships among homologous chromosomes in autotetraploids. a Coalignment of four homologous chromosome axes at mid-prophase in tetraploid onion (Allium porrum) (from Stack and Roelofs 1996). The arrows indicate early recombination nodules which mark the sites of early recombination interactions. b Immunostaining of the spread Arabidopsis arenosa tetraploid for axis component ASY1 and SC component ZYP1, showing both coalignment of all four homolog axes (left arrow) and pairwise synapsis (right arrow) (c) (C. Morgan and C.F., unpublished). c-g Quadrivalents in tetraploid Bombyx spermatocytes (from Rasmussen 1987). c-e Three examples of configurations exhibiting partial synapsis plus pre-synaptic associations

(e.g., arrow in d). f, g Two configurations exhibiting nearly complete synapsis. The four chromosomes are drawn with different colors. Note that chromosomes twist during SC formation. Quadrivalent frequencies diminish as the extent of synapsis increases such that, by the end of pachytene, the frequency of quadrivalents closely matches the frequency of chiasmata seen at metaphase I. By implication, the associations seen at the end of pachytene are stabilized by the occurrence of crossing over (or, at least, "crossover designation"), with one $\mathrm{CO}$ on each of the four arms. Once the SC disappears, these pachytene quadrivalents lead to ring quadrivalents at metaphase I

sufficiently long distance can ensure that no chromosome becomes connected to more than two partners and, more specifically, that such two-partner connections will occur near the ends of the chromosomes. These effects are illustrated below (Fig. 6b, c).

\section{Predictions}

If $\mathrm{CO}$ interference acts over a distance longer than the length of a particular chromosome, a first $\mathrm{CO}$ designation will always preclude the occurrence of a second $\mathrm{CO}$ designation anywhere else on either of the two participating homologs, even in regions where they are paired to other partners. A second $\mathrm{CO}$ designation will then occur only on one of the other two

homologs. The result would be a pair of bivalents, each linked by only a single $\mathrm{CO}$. In this situation, the COs in question could occur anywhere along the lengths of the chromosomes.

In a less extreme case, where the interference distance is somewhat less than the length of a chromosome, the possible outcomes will be bivalents, with either a single interstitial or two terminal chiasmata, and ring and chain quadrivalents linked by terminal chiasmata (Fig. 6b, c). These outcomes can be understood as follows:

(1) If the first $\mathrm{CO}$ designation occurs at an interstitial location, it will preclude $\mathrm{CO}$ designations anywhere else on the involved chromosome pair, thus ensuring that other $\mathrm{CO}$ designations involve the other pair to create a pair of 
A Hypothesis

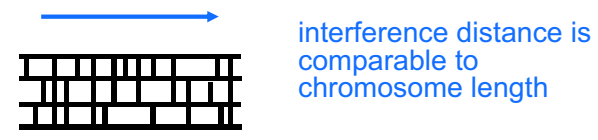

B Bivalents
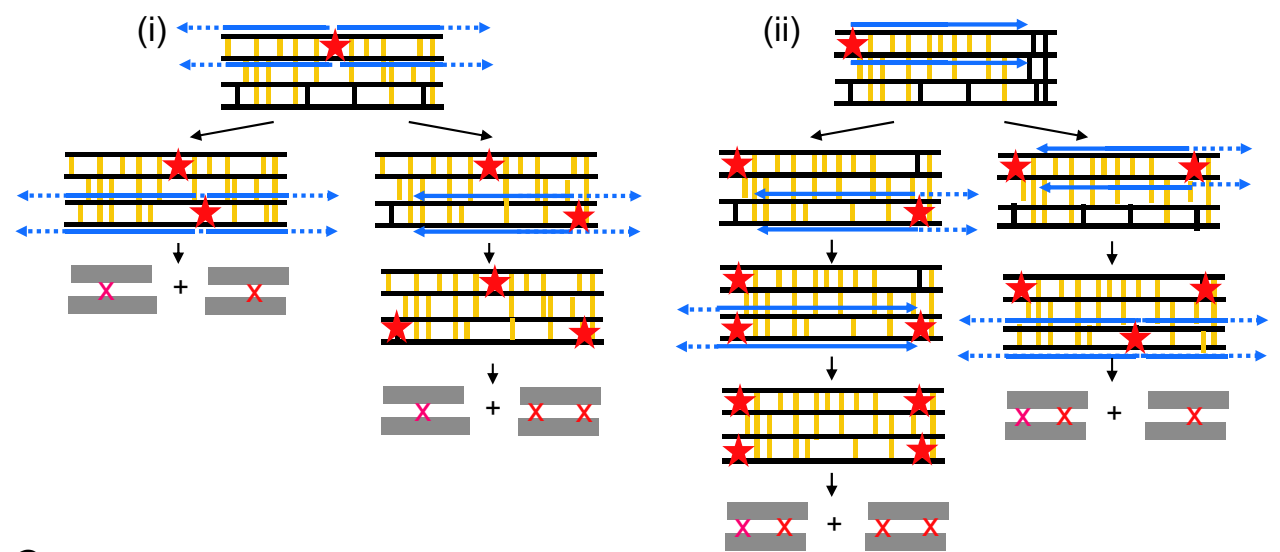

C Quadrivalents

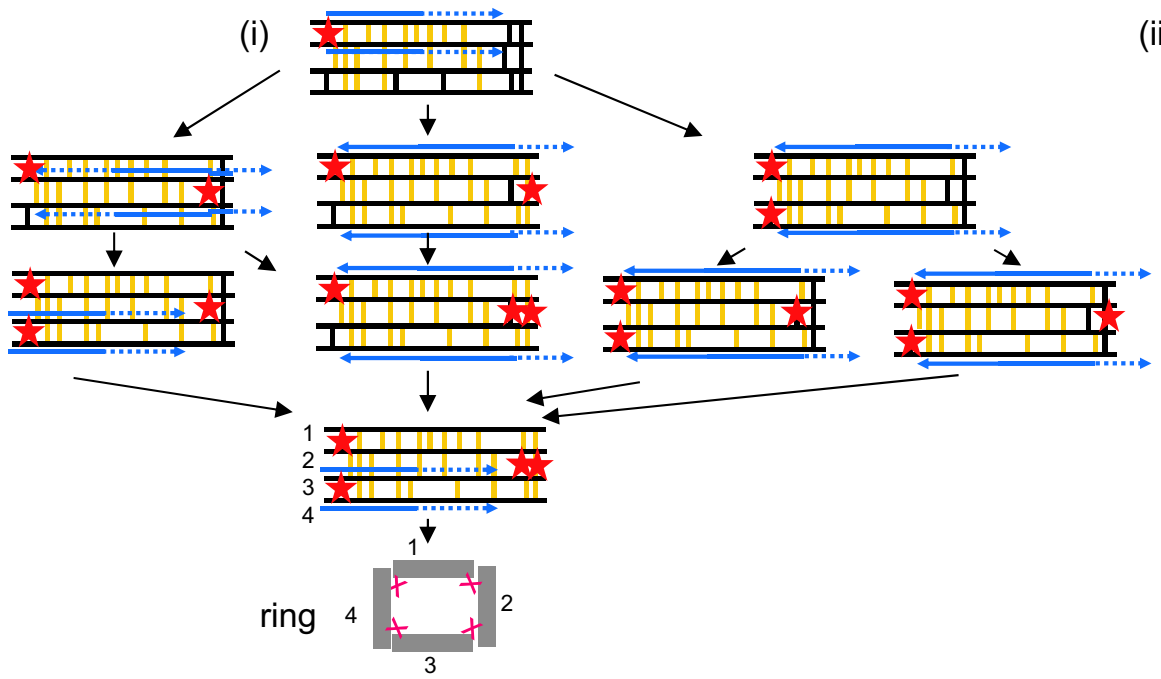

(ii)

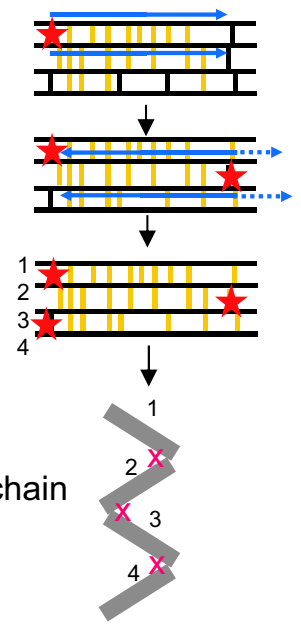

Fig. 6 Predicted chiasma/CO patterns for an evolved autotetraploid. a $\mathrm{CO}$ sites are proposed to be selected with efficient $\mathrm{CO}$ designation and accompanying $\mathrm{CO}$ interference which extends over an effective distance comparable to the length(s) of the chromosome(s) (text; representations as in Fig. 4a). If the interference distance is longer than the chromosome length, the predicted outcome is a full complement of bivalents, each with a single chiasma (not shown). b, $\mathbf{c}$ Predicted outcomes if the interference distance is somewhat less than the total chromosome length comprise a mixture of bivalents and quadrivalents, where the quadrivalents are chains or rings in which all chromosomes are linked by (sub-)terminal chiasmata. b Bivalents of different types can arise if the first chiasma is interstitial (i) or sub-terminal (ii) and according to the starting array of early recombinational interactions (not shown). c Quadrivalents can only arise if the first chiasma is sub-terminal and will comprise rings $(i)$ or chains (ii) according to the particular starting array of early recombinational interactions. In the example shown, the right-most interaction between top and bottom chromosomes is present in $i$ but absent in $i i$, thereby limiting the number of $\mathrm{CO}$ designations to 3 . Importantly, the occurrence of univalents, e.g., in trivalent-plusunivalent configurations, is precluded with efficient $\mathrm{CO}$ designation which ensures that every chromosome will experience at least one such event (text; not shown) bivalents. The second pair might also acquire a single interstitial $\mathrm{CO}$; however, if the second $\mathrm{CO}$ designation is near a chromosome end, the third $\mathrm{CO}$ designation could occur at the opposite end, generating a bivalent with a pair of terminal COs (Fig. 6b(i)).
(2) If the first $\mathrm{CO}$ designation occurs near a chromosome end, diverse patterns can arise according to the locations of the early recombination interactions and the particular sequence of subsequent $\mathrm{CO}$ designations (Fig. 6b(iii), c). Univalents are excluded by efficient 
CO designation. Thus, the only possibilities are bivalents and quadrivalents. Moreover, since an event at one end precludes the involvement of the two affected chromosomes along most of their lengths, any second $\mathrm{CO}$ designation involving one or both chromosomes of the original pair will occur at the opposite end from the first $\mathrm{CO}$ designation. Given these rules, the only possible outcome is a pair of bivalents [at least one of which has a pair of terminal chiasmata, and the other of which may be the same or may have a single interstitial chiasma (Fig. 6b(ii)); as linkages progress from chromosome to chromosome, ring and chain quadrivalents linked by terminal chiasmata (Fig. 6c)]. Chains and rings are distinguished by the particular pattern of early recombination events linking the four chromosomes prior to $\mathrm{CO}$ designation (Fig. 6c(i, ii)).

It can be noted that these outcomes can emerge from situations in which the four homologous chromosomes are linked by early recombinational interactions all along their lengths. Of course, however, if early $\mathrm{CO}$ designations occur preferentially near the chromosome ends, then outcomes involving terminal chiasmata will be more strongly favored. A recent analysis of diploid barley raises an interesting possibility of this nature (Higgins et al. 2014b). In that species, the initiation of recombination occurs along the lengths of the chromosomes but occurs much earlier near chromosome ends than in interstitial regions. Moreover, COs/chiasmata occur differentially in the terminal regions. It was suggested that this pattern could reflect the operation of $\mathrm{CO}$ interference, with early $\mathrm{CO}$ designations in terminal regions setting up interference that spreads inward, thus precluding interstitial CO designations. Importantly, by this hypothesis, terminal chiasmata are the indirect consequence of three factors: localized recombination initiation, the temporal program of $\mathrm{CO}$ designation, and $\mathrm{CO}$ interference over an appropriate distance. There would be no intrinsic local preference for $\mathrm{CO}$ designation to occur at chromosome ends per se. Operation of such a system would obviously be advantageous also for autotetraploids.

We also note that a role for interference for chiasma configurations in tetraploids has been considered by Sybenga and colleagues (e.g., Sybenga et al. 1994), although not elaborated in a context of recent advances in understanding of this process.

\section{Supporting evidence}

Several additional observations support the above scenario.

MI bivalent associations seen in well-evolved autotetraploids often involve one and only one chiasma, versus larger numbers of chiasmata per bivalent in their diploid precursors (e.g., above; Dawson 1941; Mulligan 1967; Gillies 1969;
Wolf et al. 1989; Davies et al. 1990; Carvalho et al. 2010; Yant et al. 2013).

The proposed mechanism predicts that within a given organism, when different chromosomes are of different lengths, bivalents will be more likely for shorter chromosomes whereas quadrivalents will be more likely for longer chromosomes. Correspondingly, in experimentally evolved autotetraploids of A. thaliana and natural polyploids of Zea perennis and L. corniculatus, the shorter chromosomes tend to occur as bivalents whereas the longer chromosomes tend to occur as quadrivalents with terminal associations (Dawson 1941; Shaver 1962; Davies et al. 1990; Santos et al. 2003). Interestingly, also, when chiasma configurations are analyzed as a function of evolutionary time after a de novo creation of an autotetraploid, regular segregation is achieved first for shorter chromosomes with longer chromosomes following later (Santos et al. 2003).

One way to increase the effective interference distance would be to decrease chromosome length (above). In accordance with this possibility, evolved autotetraploids in the three well-defined cases have been shown to have shorter prophase chromosomes than the diploids from which they evolved. This is true in the sand cress (A. arenosa; Higgins et al. 2014a), alfalfa (Medicago sativa; Gillies 1969), and male silk moths (Bombyx mori; Rasmussen 1987). In Bombyx, for example, the total diploid SC length per nucleus is $213 \mu \mathrm{m}$ at early pachytene and $215 \mu \mathrm{m}$ at late pachytene while the tetraploid length is $190 \mu \mathrm{m}$ at early pachytene and $186 \mu \mathrm{m}$ at late pachytene. Whether the actual interference distance (in $\mu \mathrm{m}$ ) also increases in these situations remains to be determined.

In cereals and grasses, all $\mathrm{COs} /$ chiasmata are relatively terminal. Classical studies have argued for a model in which pairs of bivalent "ends" engage in $\mathrm{CO}$ formation randomly in all combinations (the so-called "random end pairing model," where the word "pairing" was used to denote chiasma formation; Morrison and Rajhathy 1960a, b; discussion in Santos et al. 2003). The predicted proportion of bivalents versus quadrivalents in such case is $\sim 1 / 3$ versus $\sim 2 / 3$, which matches the experimental observations in these organisms (e.g., Morrison and Rajhathy 1960b). This outcome is explained explicitly by the scenario proposed above, if one further assumes a strong tendency for first $\mathrm{CO}$ designations to occur near chromosome ends (e.g., as in barley diploid meiosis; above).

\section{Other possibilities?}

What other modulations of the recombination process might allow evolution of stably fertile autotetraploid species? It might be imagined that the number of COs might be reduced in other ways. One possibility would be a reduction in the number of total recombination interactions. However, this is unlikely to be a major factor because of the phenomenon of 
CO homeostasis (Martini et al. 2006; Zhang et al. 2014b): as the number of total interactions decreases, the probability that a given interaction will be subject to $\mathrm{CO}$ interference also decreases, thereby counterbalancing the effect of fewer initial interactions. $\mathrm{CO}$ number could also be decreased in two other ways: first, the efficiency of $\mathrm{CO}$ designation could be reduced, and second, $\mathrm{CO}$-designated interactions might not all mature into COs (Zhang et al. 2014a). Such changes might result, for example, from hypomorphic alterations in molecules involved specifically in the $\mathrm{CO}$ formation process. However, all of these possible effects also increase the probability that some chromosome pairs will not acquire even a single interaction and thus will not acquire even a single $\mathrm{CO}$ and thus, potentially, the frequency of univalents (Zhang et al. 2014a). In contrast, no such risk is conferred by reducing the $\mathrm{CO}$ number by increasing the interference distance. Additionally, these types of changes will reduce the $\mathrm{CO}$ formation randomly along the chromosomes and thus cannot explain changes in CO positioning.

For species that exhibit mostly terminal COs, it is alternatively possible to envision that $\mathrm{CO}$ designation might be intrinsically limited to regions near chromosome ends, irrespective of interference, either because early interactions are limited to these regions or because the $\mathrm{CO}$ designation probability is much higher in these regions. In either of these cases, and assuming that $\mathrm{CO}$ designation is very efficient, random $\mathrm{CO}$ designation at pairs of ends, in all possible combinations, could have the same effect as the model proposed here, e.g., for cereals and grasses as discussed above. However, such a model cannot explain cases in which other patterns are observed, e.g., when interstitial chiasmata are common or others. In contrast, the proposed model can synthetically explain diverse observed patterns.

It is also possible that a significant role is played by events during early prophase by which chromosomes first choose partners, i.e., by partner interactions even before DSB formation ("DSB-independent pairing") or during DSB-mediated coalignment process prior and prerequisite to $\mathrm{CO}$ designation. This may seem unlikely in view of the complex patterns of associations that are seen at leptotene/ zygotene in evolved tetraploid lines as well as their newly formed counterparts (e.g., Fig. 5a, b; Hobolth 1981; Gillies et al. 1987; Rasmussen 1987; Davies et al. 1990; Carvalho et al. 2010). On the other hand, in a Sordaria diploid, a mutation that permits DSB formation but delays coalignment also results in massive chromosome entanglements, implying that DSB-mediated coalignment at one position tends to promote subsequent coalignment events at adjacent positions, thus drawing the pair of partners out of the "pairing pool" (Storlazzi et al. 2010). Operation of such an effect in a tetraploid would tend to promote simpler arrays of coalignment associations and, thus, bivalent formation versus formation of multivalents.
Similarly, homology-based pairwise associations in centromere regions (e.g., within non-specific centromere clusters) or early pairwise DSB-independent pairing of telomeres (review in Zickler and Kleckner 2015; Higgins et al. 2014a) might propagate along the corresponding chromosome arms, by independent pairing and/or DSBmediated coalignment, again simplifying partner associations and, thus, CO patterns. Simplification at this early stage has a particular advantage that it would affect not only the pairwise connections resulting from COs that are subject to interference but also those resulting from the significant minority of COs that occur without being subject to CO interference (e.g., Mercier et al. 2015).

Another interesting point is that, since the two genomes of a diploid are never perfectly identical, whole genome duplication, e.g., by colchicine treatment, may produce an autotetraploid comprising two pairs of genomes where the members of each pair are identical while the members of different pairs have slight differences. Such pair-to-pair differences play an obvious determining role in evolved allopolyploids where different pairs of closely related homologs are more different from one another and, in consequence, crossovers occur preferentially between the members of each closely related pair (e.g., Holm 1986; Martín et al. 2014). The same effects could potentially be subtly significant in autotetraploids. However, if so, their effects may not be significant beyond early generations, given that evolved autotetraploids generally show tetrasomic inheritance.

\section{Elimination of interlockings?}

Restriction of the number and/or positions of $\mathrm{CO}$ designations could potentially be sufficient to eliminate the interlock problem. Interlocks arise during the DSB-mediated coalignment of homologs and, thus, prior CO designation (von Wettstein et al. 1984; Storlazzi et al. 2010). Thus, changes in CO patterns are unlikely to alter the probability with which interlocks form. In contrast, simplification of $\mathrm{CO}$ patterns might well facilitate the resolution of interlocks after they have formed. For this to be true, $\mathrm{CO}$ interactions would have to comprise the only effective linkages between homologous chromosomes and, thus, the only linkages that are preventing entangled chromosomes from assuming a regular relationship. Available evidence is consistent with this possibility. (i) Recombinational interactions that are not CO fated have progressed to the "non-crossover fate," and the corresponding complexes are lost from the chromosome axes. (ii) Despite the fact that $\mathrm{SC}$ is forming during this period, SC patterns are known to undergo adjustment to give final configurations in which $\mathrm{SC}$ segments correspond to $\mathrm{CO}$ positions (e.g., in tetraploid Bombyx; Fig. 5f, g). Thus, apparently, $\mathrm{SC}$ is destabilized except at $\mathrm{CO}$ sites and reforms outward from those sites (which also appears to occur in allohexaploid wheat; discussion in Zickler and Kleckner 1999). 
Correspondingly, SC may be a permanent impediment to interlock resolution only at positions of COs, rather than globally.

\section{Molecular basis of evolved autotetraploidy}

Recent studies provide insights into which genes came under natural selection during adaptation to whole genome duplication in a natural autopolyploid, A. arenosa (Hollister et al. 2012; Yant et al. 2013). A. arenosa has both diploid populations and a natural autotetraploid variant that is at least 15 , 000 years old (Arnold et al. 2015). This autotetraploid is meiotically stable and fully fertile. Also, it exhibits features that are well explained by our proposed model. All four copies of each chromosome clearly coalign and associate in zygotene and pachytene (Carvalho et al. 2010; Higgins et al. 2014a), and yet, at metaphase I, almost only bivalents are present. Further, the autotetraploid form has lower chiasma formation rates than the diploid, usually only forming one chiasma per bivalent (Comai et al. 2003; Carvalho et al. 2010; Yant et al. 2013; Higgins et al. 2014a; Fig. 1(c), right). Moreover, the axes in evolved autotetraploid lines are 5-10\% shorter than axes in closely related diploids, implying a corresponding increase in the effective interference distance as explained above (Higgins et al. 2014a; C. Morgan, C. Franklin, and K. B., unpublished).

Additional studies provide insight into which specific molecules might be involved in stabilization of autotetraploidy in A. arenosa. A genome scan comparing diploid and tetraploid lines showed strong evidence of selection having acted on eight genes whose functions are exerted specifically during meiosis (Hollister et al. 2012; Yant et al. 2013). The proteins encoded by these genes include chromosome axis components ASY1 and ASY3 (homologs of budding yeast Hop1 and Red1), cohesins and cohesin-associated proteins SMC3 and SYN1 (Rec8) and PDS5, and synaptonemal complex transverse filament proteins ZYP1a and ZYP1b (encoded by two tandemly duplicated genes). Mutant studies in closely related $A$. thaliana have demonstrated that mutation of these genes results in defects in recombination, including $\mathrm{CO}$ formation (Bai et al. 1999; Armstrong 2002; Higgins et al. 2005; De Muyt et al. 2009; Ferdous et al. 2012). In contrast, while the alleles selected in tetraploid $A$. arenosa carry mutations that have the potential to alter protein function or form, they do not appear to be loss-of-function mutations (Yant et al. 2013; Wright et al. 2015).

It is intriguing that evolution of stable autotetraploidy involves these key axis components. Alterations in axis components are known to alter the strength of $\mathrm{CO}$ interference (Zhang et al. 2014b; Libuda et al. 2013) and also can alter axis length (Revenkova et al. 2004; Novak et al. 2008), which will alter the effective $\mathrm{CO}$ interference distance as a fraction of physical and genomic chromosome length (Zhang et al. 2014b) as described above.

\section{Summary}

How can a newly formed autotetraploid evolve to a stable sexually reproducing state, with a concomitant modulation of chiasma number and pattern? This question has been discussed for a century or more. Recent progress in the understanding and analysis of meiotic recombination opens the way to considering specific proposals for a mechanism as well as new experimental approaches. We focus here on the possibility that modulation of crossover interference, either directly and/or indirectly via changes in axis length, could play a central role. Our proposal explains the patterns of COs/chiasmata observed for species of evolved autotetraploids that exhibit primarily bivalents and for species that exhibit both bivalents and quadrivalents as well as accommodating the tendency, seen in several cases, for COs/chiasmata to occur near chromosome ends. It may also explain the fact that evolved autotetraploids lack interchromosomal interlocks characteristic of neo-autotetraploid lines. Evaluation of this and other proposals by application of molecular methodologies provides a fertile ground for future studies.

Acknowledgments We gratefully acknowledge Chris Morgan for providing unpublished images and Jim Henle for helping in manuscript preparation. The authors' research on meiosis is funded by grants to NK (NIH R01 GM044794), DZ (Centre National de la Recherche Scientifique (Unité Mixte de Recherche 8621)), and CF (Biotechnology and Biological Sciences Research Council, UK, grants BB/M004902/1). We also appreciate the careful consideration of the manuscript by the reviewers and their suggestions as to potential contributions of early (pre-CO) pairing/coalignment interactions and of low-level DNA sequence differences between homologs.

Compliance with ethical standards This article does not contain any studies with human participants or animals performed by any of the authors.

Conflict of interest The authors declare that they have no competing interests.

Open Access This article is distributed under the terms of the Creative Commons Attribution 4.0 International License (http:// creativecommons.org/licenses/by/4.0/), which permits unrestricted use, distribution, and reproduction in any medium, provided you give appropriate credit to the original author(s) and the source, provide a link to the Creative Commons license, and indicate if changes were made.

\section{References}

Albini SM, Jones GH (1987) Synaptonemal complex spreading in Allium cepa and A. fistulosum. I. The initiation and sequence of pairing. Chromosoma 95:324-338 
Armstrong SJ (2002) Asy1, a protein required for meiotic chromosome synapsis, localizes to axis-associated chromatin in Arabidopsis and Brassica. J Cell Sci 115:3645-3655. doi:10.1242/jcs.00048

Arnold B, Kim ST, Bomblies K (2015) Single geographic origin of a widespread autotetraploid Arabidopsis arenosa lineage followed by interploidy admixture. Mol Biol Evol 32:1382-1395

Arrigo N, Barker MS (2012) Rarely successful polyploids and their legacy in plant genomes. Curr Opin Plant Biol 15:140-146

Astaurov BL (1969) Experimental polyploidy in animals. Annu Rev Genet 3:99-126. doi:10.1146/annurev.ge.03.120169.000531

Bai X, Peirson BN, Dong F et al (1999) Isolation and characterization of SYN1, a RAD21-like gene essential for meiosis in Arabidopsis. Plant Cell 11:417-430

Bhullar R, Nagarajan R, Bennypaul H, Sidhu GK, Sidhu G, Rustgi S, von Wettstein D, Gill KS (2014) Silencing of a metaphase I-specific gene results in a phenotype similar to that of the pairing homeologous 1 (Ph1) gene mutations. Proc Natl Acad Sci USA 111(39):1418714192

Bomblies K, Madlung A (2014) Polyploidy in the Arabidopsis genus. Chromosome Res 22:117-134. doi:10.1007/s10577-014-9416-x

Bremer G, Bremer-Reinders DE (1954) Breeding of tetraploid rye in the Netherlands. I. Methods and cytological observations. Euphytica 3: 49-63

Carvalho A, Delgado M, Barão A et al (2010) Chromosome and DNA methylation dynamics during meiosis in the autotetraploid Arabidopsis arenosa. Sex Plant Reprod 23:29-37. doi:10.1007/ s00497-009-0115-2

Comai L (2005) The advantages and disadvantages of being polyploid. Nat Rev Genet 6:836-846. doi:10.1038/nrg1711

Comai L, Tyagi AP, Lysak MA (2003) FISH analysis of meiosis in Arabidopsis allopolyploids. Chromosome Res 11:217-226

Davies A, Jenkins G, Rees H (1990) Diploidization of Lotus corniculatus L. (Fabaceae) by elimination of multivalents. Chromosoma 99:289 295

Dawson C (1941) Tetrasomic inheritance in Lotus corniculatus L. J Genet 42:49-73

De Muyt A, Pereira L, Vezon D et al (2009) A high throughput genetic screen identifies new early meiotic recombination functions in Arabidopsis thaliana. PLoS Genet 5:e1000654. doi:10.1371/ journal.pgen.1000654

Doyle JJ, Flagel LE, Paterson AH et al (2008) Evolutionary genetics of genome merger and doubling in plants. Annu Rev Genet 42:443461. doi:10.1146/annurev.genet.42.110807.091524

Fawcett JA, Van de Peer Y (2010) Angiosperm polyploids and their road to evolutionary success. Trends Evol Biol 2:3. doi:10. 4081/eb.2010.e3

Ferdous M, Higgins JD, Osman K et al (2012) Interhomolog crossingover and synapsis in Arabidopsis meiosis are dependent on the chromosome axis protein AtASY3. PLoS Genet 8:e1002507

Fjellstrom RG, Beuselinck PR, Steiner JJ (2001) RFLP marker analysis supports tetrasomic inheritance in Lotus corniculatus L. Theor Appl Genet 102:718-725

Fox DP (1973) The control of chiasma distribution in the locust Schistocerca gregaria (Forskal). Chromosoma 43:289-328

Gilles A, Randolph LF (1951) Reduction of quadrivalent frequency in autotetraploid maize during a period of ten years. Am J Bot 38:1217

Gillies CB (1969) Alfalfa chromosomes. II. Pachytene karyotype of a tetraploid Medicago sativa L. Crop Science 10:172-175

Gillies CB, Kuspira J, Bhambhani RN (1987) Genetic and cytogenetic analyses of the A genome of Triticum monococcum. IV. Synaptonemal complex formation in autotetraploids. Genome 29: 309-318

Grandont L, Jenczewski E, Lloyd A (2013) Meiosis and its deviations in polyploid plants. Cytogenet Genome Res 140:171-184. doi:10. $1159 / 000351730$
Gruhn JR, Rubio C, Broman KW, Hunt PA, Hassold T (2013) Cytological studies of human meiosis: sex-specific differences in recombination originate at, or prior to, establishment of doublestrand breaks. PLoS One 8(12):e85075. doi:10.1371/journal.pone. 0085075

Hazarika MH, Rees H (1967) Genotypic control of chromosome behaviour in rye. X. Chromosome pairing and fertility in autotetraploids. Heredity 22:317-322. doi:10.1038/hdy.1967.44

Higgins JD, Sanchez-Moran E, Armstrong SJ et al (2005) The Arabidopsis synaptonemal complex protein ZYP1 is required for chromosome synapsis and normal fidelity of crossing over. Genes Dev 19:2488-2500. doi:10.1101/gad.354705

Higgins JD, Osman K, Jones GH, Franklin FC (2014a) Factors underlying restricted crossover localization in barley meiosis. Annu Rev Genet 48:29-47

Higgins JD, Wright KM, Bomblies K, Franklin FCH (2014b) Cytological techniques to analyze meiosis in Arabidopsis arenosa for investigating adaptation to polyploidy. Front Plant Sci 4:546. doi:10.3389/ fpls.2013.00546

Hillers KJ, Villeneuve AM (2003) Chromosome-wide control of meiotic crossing over in C. elegans. Curr Biol 13:1641-1647

Hilpert G (1957) Effect of selection for meiotic behaviour in autotetraploid rye. Hereditas 43:318-322

Hobolth P (1981) Chromosome pairing in allohexaploid wheat var. Chinese Spring. Transformation of multivalents into bivalents, a mechanism for exclusive bivalent formation. Carlsberg Res Commun 46:129-173

Hollister JD, Arnold BJ, Svedin E et al (2012) Genetic adaptation associated with genome-doubling in autotetraploid Arabidopsis arenosa. PLoS Genet 8:e1003093. doi:10.1371/journal.pgen.1003093

Holm PB (1986) Chromosome pairing and chiasma formation in allohexaploid wheat, Triticum aestivum analyzed by spreading of meiotic nuclei. Carlsberg Res Commun 51:239-294

Hunter N (2006) Meiotic recombination. In: Aguilera A, Rothstein R (eds) Molecular genetics of recombination. Topics in current genetics. Springer, Heidelberg, pp 381-442

Ianzini F, Kosmacek EA, Nelson ES et al (2009) Activation of meiosisspecific genes is associated with depolyploidization of human tumor cells following radiation-induced mitotic catastrophe. Cancer Res 69:2296-2304. doi:10.1158/0008-5472.CAN-08-3364

Jones GH (1967) The control of chiasma distribution in rye. Chromosoma 22:69-90. doi:10.1007/BF00291287

Jones GH, Vincent JE (1994) Meiosis in allopolyploid Crepis capillaris. II. Autotetraploids. Genome 37:497-505

Kleckner N (2006) Chiasma formation: chromatin/axis interplay and the role(s) of the synaptonemal complex. Chromosoma 115:175-194

Kleckner N, Zickler D, Jones GH, Dekker J, Padmore R, Henle J, Hutchinson J (2004) A mechanical basis for chromosome function. Proc Nat Acad Sci USA 101:12592-12597

Kleckner N, Zhang L, Weiner B and Zickler D (2011) Meiotic chromosome dynamics in genome organization and function in the cell nucleus. ed. K. Rippe. Wiley-VCH Verlag GmbH \& Co. KGaA. doi: 10.1002/9783527639991.ch19

Krebs SL, Hancock JF (1989) Tetrasomic inheritance of isoenzyme markers in the highbush blueberry Vaccinium corymbosum L. Heredity 63:11-18

Lampson MA, Cheeseman IM (2011) Sensing centromere tension: Aurora $\mathrm{B}$ and the regulation of kinetochore function. Trends Cell Biol 21:133-140

Lavania UC, Srivastava S, Sybenga J (1991) Cytogenetics of fertility improvement in artificial autotetraploids of Hyoscyamus niger $\mathrm{L}$. Genome 34:190-194

Li X, Nicklas RB (1997) Tension-sensitive kinetochore phosphorylation and the chromosome distribution checkpoint in praying mantid spermatocytes. J Cell Sci 110:537-545 
Libuda DE, Uzawa S, Meyer BJ, Villeneuve AM (2013) Meiotic chromosome structures constrain and respond to designation of crossover sites. Nature 502:703-706

López E, Pradillo M, Oliver C, Romero C, Cuñado N, Santos JL (2012) Looking for natural variation in chiasma frequency in Arabidopsis thaliana. J Exp Bot 63:887-94. doi:10.1093/jxb/err319

Martín AC, Shaw P, Phillips D, Reader S, Moore G (2014) Licensing MLH1 sites for crossover during meiosis. Nature Commun 5:4580. doi: $10.1038 /$ ncomms 5580

Martinez-Perez E, Colaiacovo MP (2009) Distribution of meiotic recombination events: talking to your neighbors. Curr Opin Genet Dev 19: 105-112. doi:10.1016/j.gde.2009.02.005

Martini E, Diaz RL, Hunter N, Keeney S (2006) Crossover homeostasis in yeast meiosis. Cell 126:285-295

McCollum CD (1958) Comparative studies of chromosome pairing in natural and induced tetraploid Dactylis. Chromosoma 9:571-605

Mercier R, Mezard C, Jenczewski E, Macaisne N, Grelon M (2015) The molecular biology of meiosis in plants. Annu Rev Plant Biol 66: 297-327. doi:10.1146/annrev-arplant-050213-035923

Mitchell-Olds T, Clauss MJ (2002) Plant evolutionary genomics. Curr Opin Plant Biol 1:74-79

Morrison JW, Rajhathy T (1960a) Chromosome behaviour in autotetraploid cereals and grasses. Chromosoma 11:297-309

Morrison JW, Rajhathy T (1960b) Frequency of quadrivalents in autotetraploid plants. Nature 187:528-530

Mosquin T (1967) Evidence for autopolyploidy in Epilobium angustifolium (Onagraceae). Evolution 21:713-719

Mulligan A (1967) Diploid and tetraploid Physaria vitulifera (Cruciferae). Can J Bot 45:183-188

Myers WM (1945) Meiosis in autotetraploid Lolium perenne in relation to chromosomal behavior in autopolyploids. Bot Gaz 106:304-316

Nicklas RB (1997) How cells get the right chromosomes. Science 275: 632-637

Novak I, Wang H, Revenkova E, Jessberger R, Scherthan H, Höög C (2008) Cohesin Smclbeta determines meiotic chromatin axis loop organization. J Cell Biol 180:83-90. doi:10.1083/jcb.200706136

Oliver-Bonet M, Campillo M, Turek PJ, Ko E, Martin RH (2007) Analysis of replication protein A (RPA) in human spermatogenesis. Mol Human Reprod 13:837-844

Otto SP, Whitton J (2000) Polyploid incidence and evolution. Annu Rev Genet 34:401-437. doi:10.1146/annurev.genet.34.1.401

Povilaitis B, Boyes JW (1956) A cytological study of autotetraploid red clover. Am J Bot 43:169. doi:10.2307/2438673

Quiros CF (1982) Tetrasomic segregation for multiple alleles in alfalfa. Genetics 101:117-127

Ramsey J, Schemske D (1998) Pathways, mechanisms, and rates of polyploid formation in flowering plants. Annu Rev Ecol Syst 29:467501

Ramsey J, Schemske D (2002) Neopolyploidy in flowering plants. Annu Rev Ecol Syst 33:589-639

Rasmussen SW (1987) Chromosome pairing in autotetraploid Bombyx males. Inhibition of multivalent correction by crossing over. Carlsberg Res Commun 52:211-242

Reddi VR (1970) Pachytene pairing and the nature of polyploidy in Sorghum arundinaceum. Caryologia 23:295-302

Revenkova E, Eijpe M, Heyting C, Hodges CA, Hunt PA, Liebe B, Scherthan H, Jessberger R (2004) Cohesin SMC1 beta is required for meiotic chromosome dynamics, sister chromatid cohesion and DNA recombination. Nat Cell Biol 6:555-562

Rieseberg L, Willis J (2007) Plant speciation. Science 317:910-914

Sanchez Moran E, Armstrong SJ, Santos JL, Franklin FC, Jones GH (2001) Chiasma formation in Arabidopsis thaliana accession
Wassileskija and in two meiotic mutants. Chromosome Res 9(2): $121-128$

Santos JL, Alfaro D, Sanchez-Moran E, Armstrong SJ, Franklin FHC, Jones GH (2003) Partial diploidization of meiosis in autotetraploid Arabidopsis thaliana. Genetics 165:1533-1540

Shaver DL (1962) A study of meiosis in perennial teosinte, in tetraploid maize and in their tetraploid hybrid. Caryologia 15:43-57

Soltis DE, Soltis PS, Tate JA (2003) Advances in the study of polyploidy since plant speciation. New Phytologist 161:173-191

Soltis DE, Soltis PS, Schemske DW et al (2007) Autopolyploidy in angiosperms: have we grossly underestimated the number of species? Taxon 1:13-30. doi:10.2307/25065732

Stack SM, Roelofs D (1996) Localized chiasmata and meiotic nodules in the tetraploid onion Allium porrum. Genome 39:770-783

Stenberg P, Saura A (2013) Meiosis and its deviations in polyploid animals. Cytogenet Genome Res 140:185-203. doi:10.1159/ 000351731

Storlazzi A, Gargano S, Ruprich-Robert G, Falque M, David M, Kleckner N, Zickler D (2010) Recombination proteins mediate meiotic spatial chromosome organization and pairing. Cell 141:94-106. doi:10. 1016/j.cell.2010.02.041

Sturtevant AH (1915) The behavior of the chromosomes as studied through linkage. Z indukt Abstamm-u VererbLehre 13:234-287

Sybenga J (1975) Meiotic configurations: a source of information for estimating genetic parameters. Springer, Berlin

Sybenga J, Schabbink E, Eden J, Jong JH (1994) Pachytene pairing and metaphase I configurations in a tetraploid somatic Lycopersicon esculentum x L. peruvianum hybrid. Genome 37(1):54-60

von Wettstein D, Rasmussen SW, Holm PB (1984) The synaptonemal complex in genetic segregation. Ann Rev Genet 18:331-413

Wang S, Zickler D, Kleckner N, Zhang L (2015) Meiotic crossover patterns: obligatory crossover, interference and homeostasis in a single process. Cell Cycle 14:305-314. doi:10.4161/15384101.2014. 991185

Wolf PG, Soltis PS, Soltis DE (1989) Tetrasomic inheritance and chromosome pairing behaviour in the naturally occurring autotetraploid Heuchera grossulariifolia (Saxifragaceae). Genome 32:655-659. doi:10.1139/g89-494

Wright KM, Arnold B, Xue K et al (2015) Selection on meiosis genes in diploid and tetraploid Arabidopsis arenosa. Mol Biol Evol 32:944 955. doi:10.1093/molbev/msu398

Wu J-H, Datson PM, Manako KI, Murray BG (2013) Meiotic chromosome pairing behaviour of natural tetraploids and induced autotetraploids of Actinidia chinensis. Theor Appl Genet 127:549-557. doi: 10.1007/s00122-013-2238-y

Yant L, Hollister JD, Wright KM et al (2013) Meiotic adaptation to genome duplication in Arabidopsis arenosa. Curr Biol 23:21512156. doi:10.1016/j.cub.2013.08.059

Zhang L, Liang Z, Hutchinson J, Kleckner N (2014a) Crossover patterning by the beam-film model: analysis and implications. PLoS Genet 10(1):e1004042. doi:10.1371/journal.pgen. 1004042

Zhang L, Wang S, Yin S, Hong S, Kim KP, Kleckner N (2014b) Topoisomerase II mediates meiotic crossover interference. Nature 511:551-556. doi:10.1038/nature13442

Zhang L, Espagne E, de Muyt A, Zickler D, Kleckner N (2015) Interference-mediated synaptonemal complex formation with embedded crossover designation. Proc Nat Acad Sci USA 111: E5059-5068. doi:10.1073/pnas.1416411111

Zickler D, Kleckner N (1999) Meiotic chromosomes: integrating structure and function. Ann Rev Genet 33:603-754

Zickler D, Kleckner N (2015) Recombination, pairing and synapsis of homologs during meiosis. Cold Spring Harb Perspect Biol 18(7):6. doi:10.1101/cshperspect.a016626 